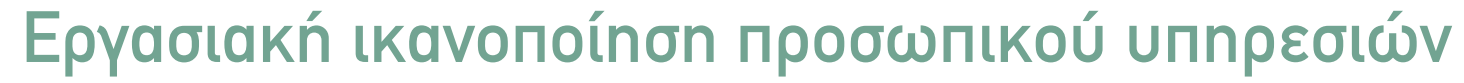

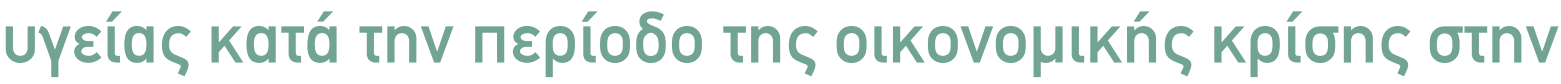

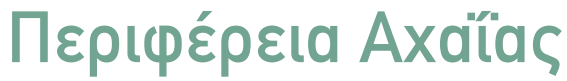

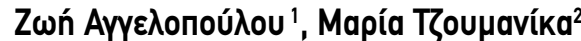

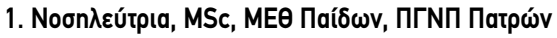

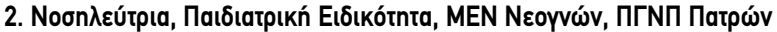

\section{ПEPIАHШH}

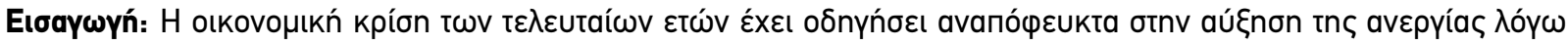

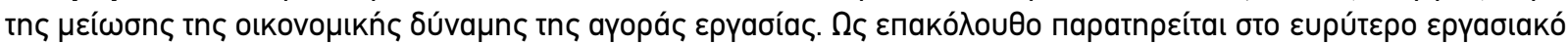

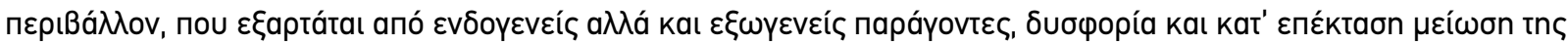

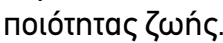

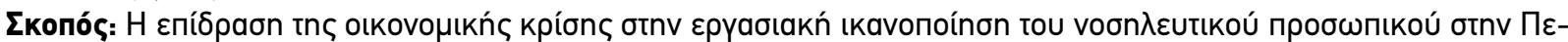

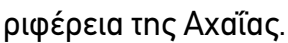

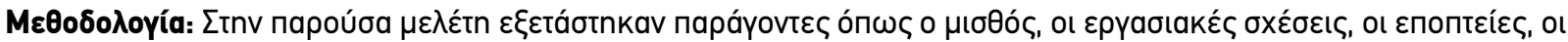

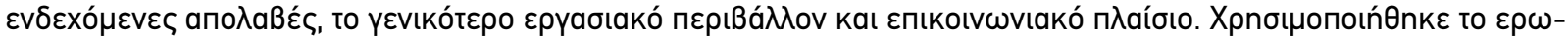

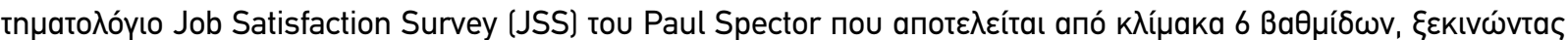

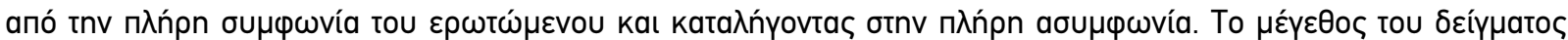

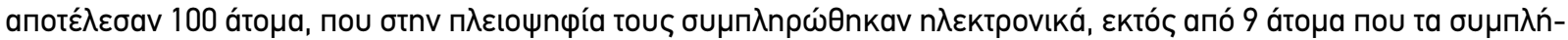

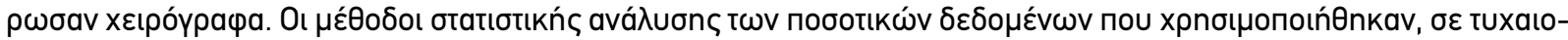

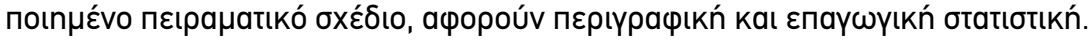

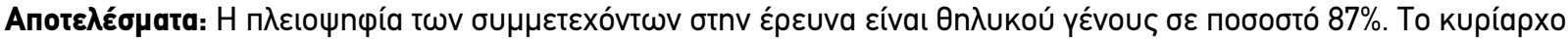

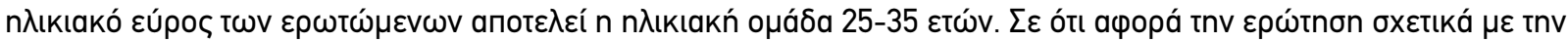

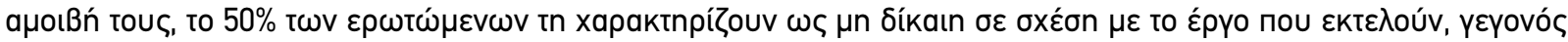

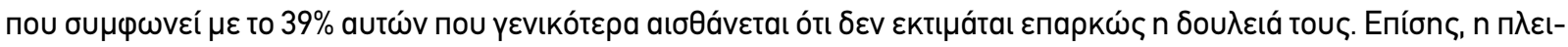

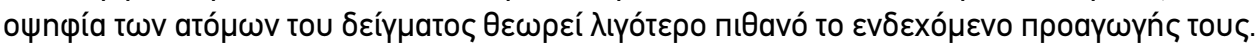

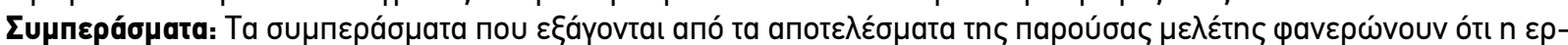

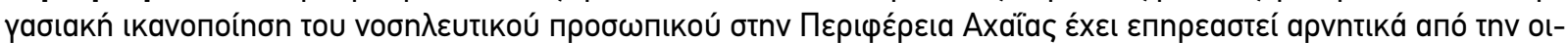

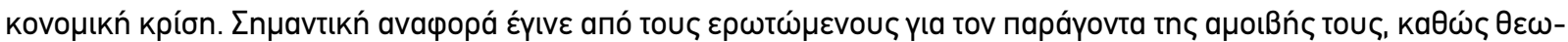

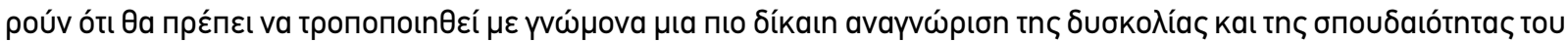

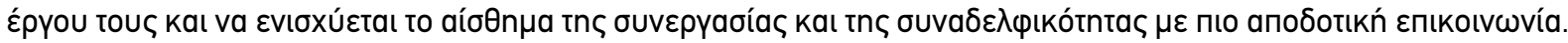

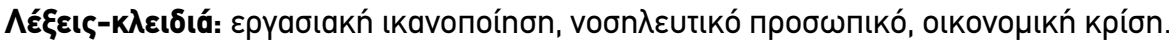

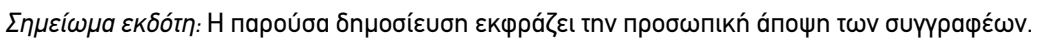




\section{EPEYNHTIKA APOPA}

\section{BAEIKA EHMEIA}

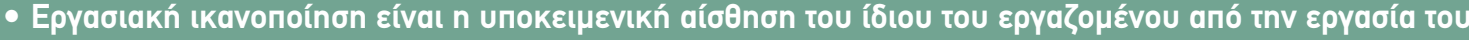

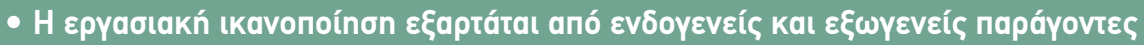

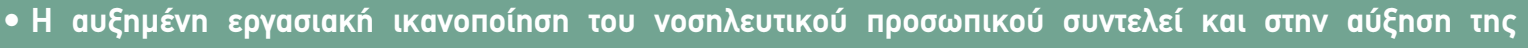

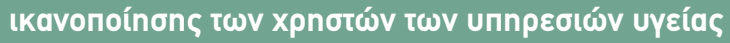

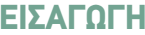

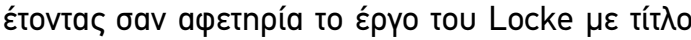

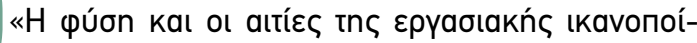

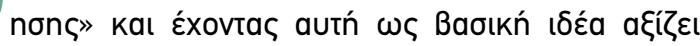

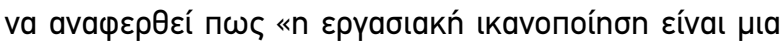

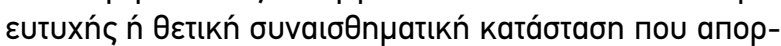

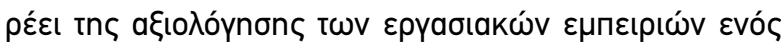

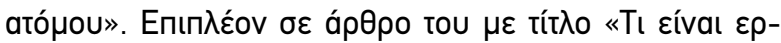

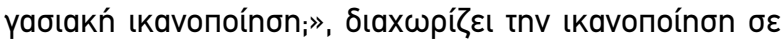

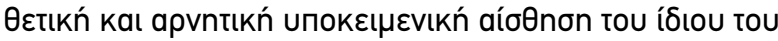

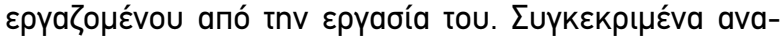

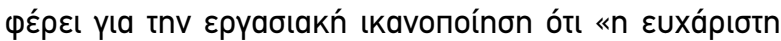

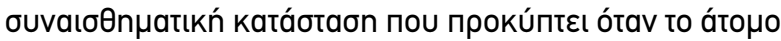

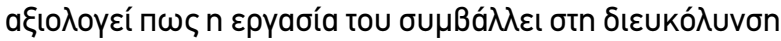

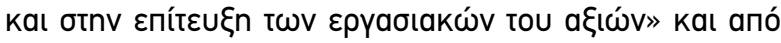

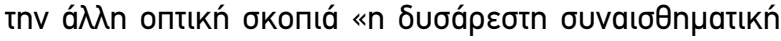

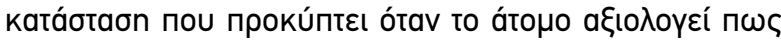

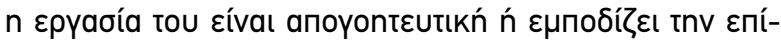

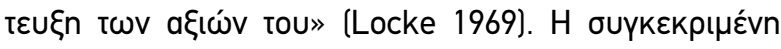

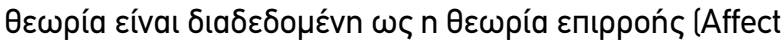

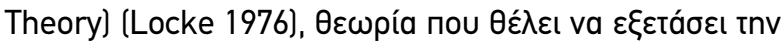

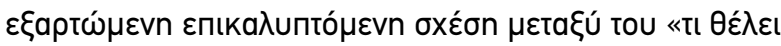

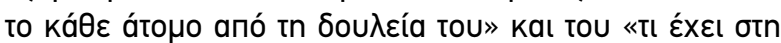

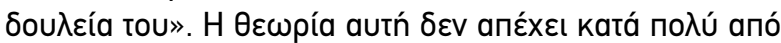

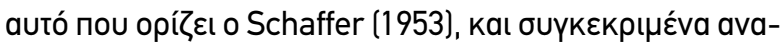

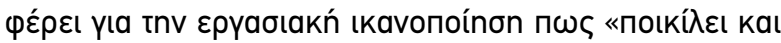

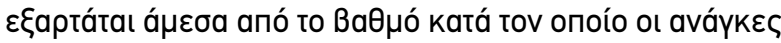

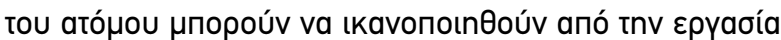

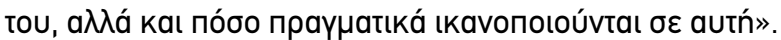

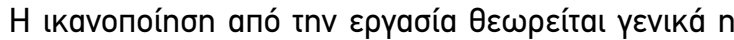

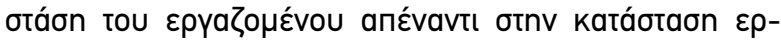

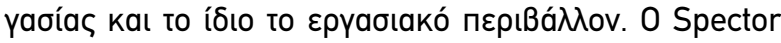

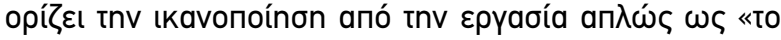

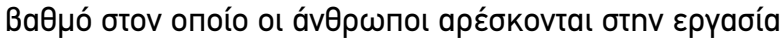

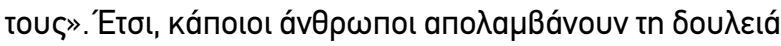

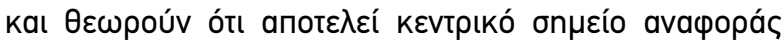

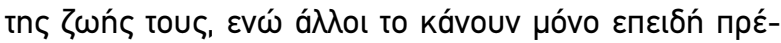

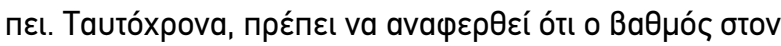

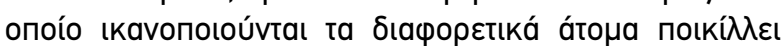

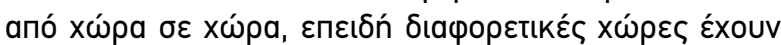

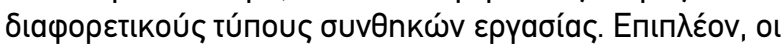

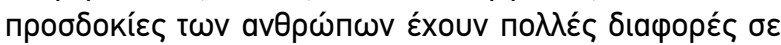

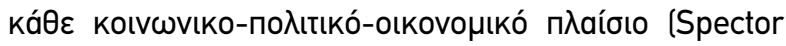

1997).

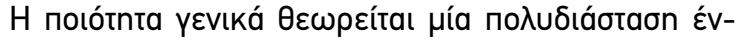

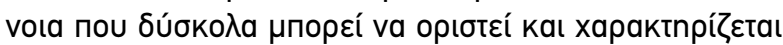

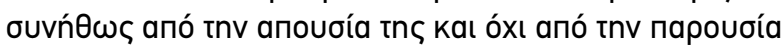

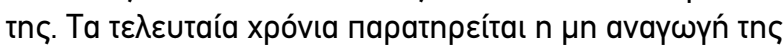

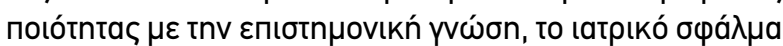

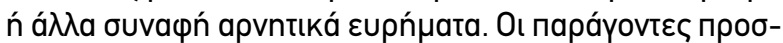

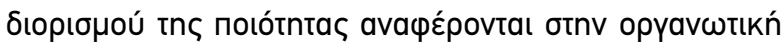

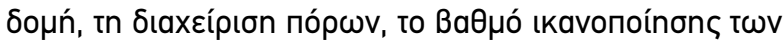

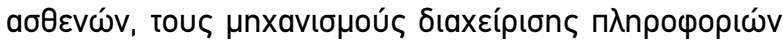

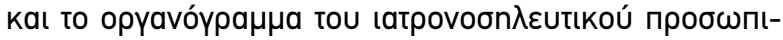
koú (Пauגáknc 2014).

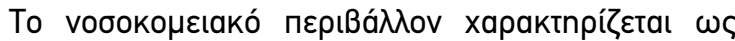

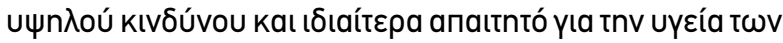

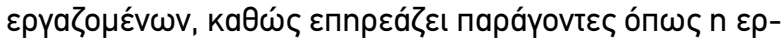

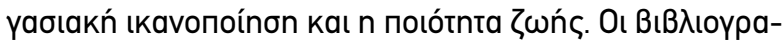

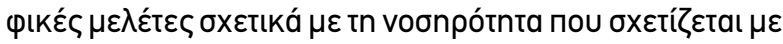

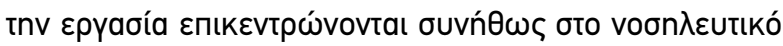

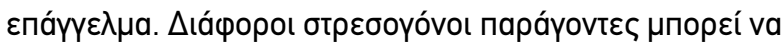

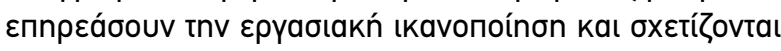

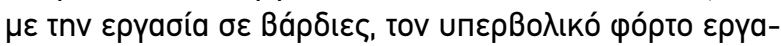

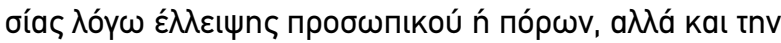

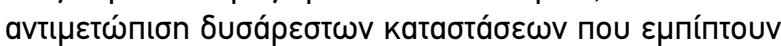

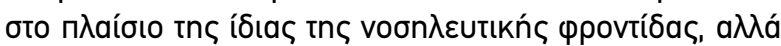

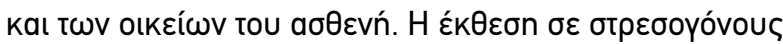

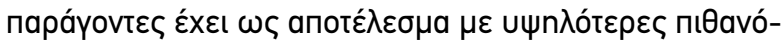

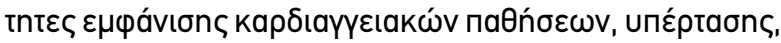

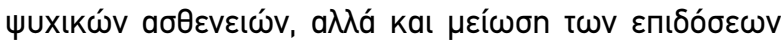

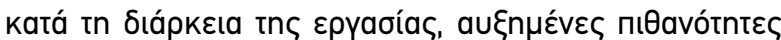

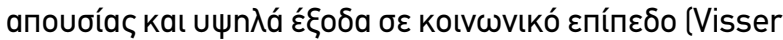
et al 2003, Faragher et al 2005, Jelastopulu et al 2013).

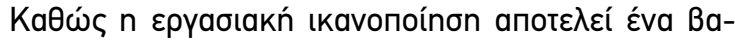

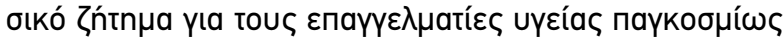

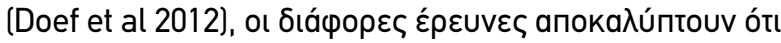

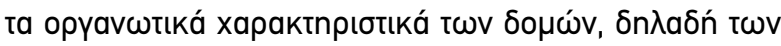

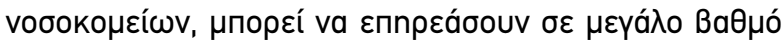

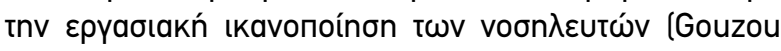

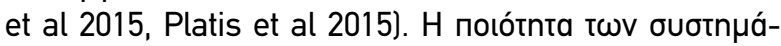

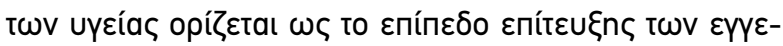

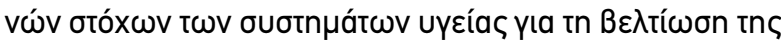

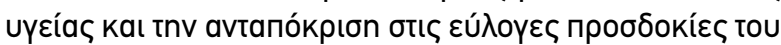

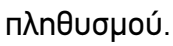

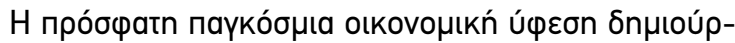




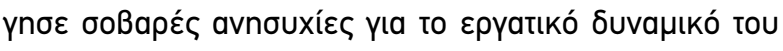

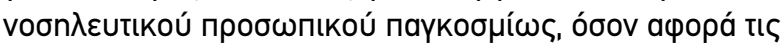

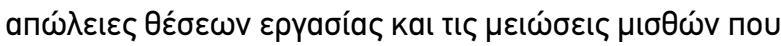

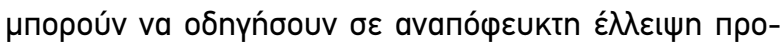

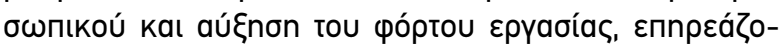

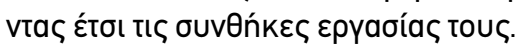

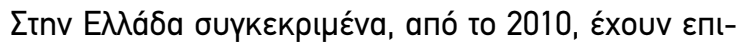

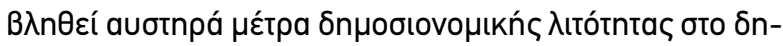

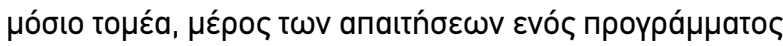

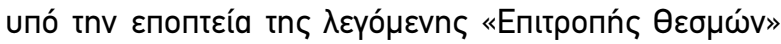

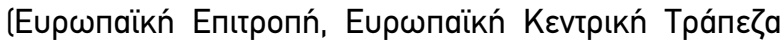

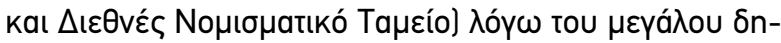

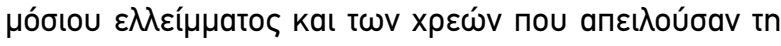

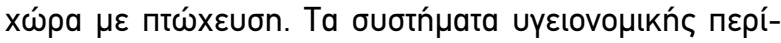

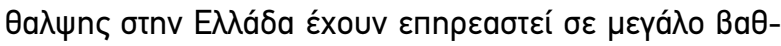

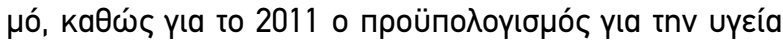

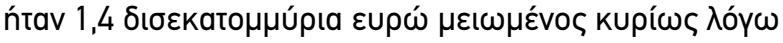

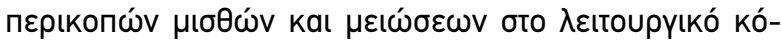

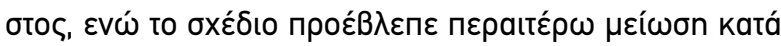

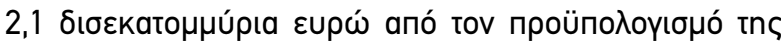

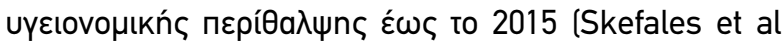

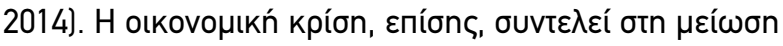

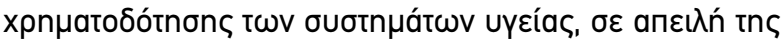

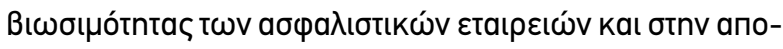

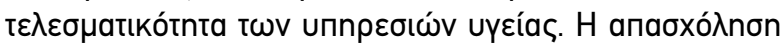

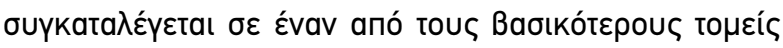

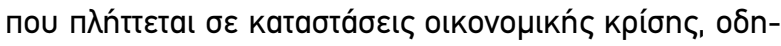

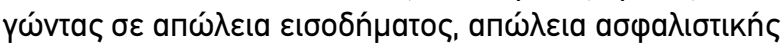

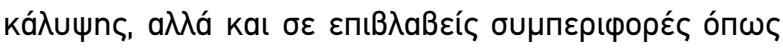

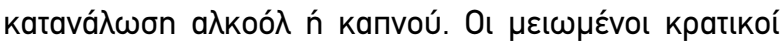

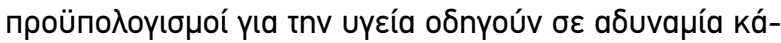

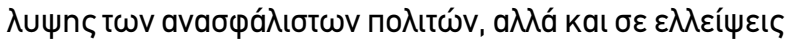

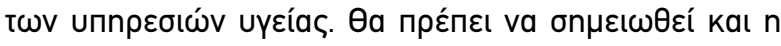

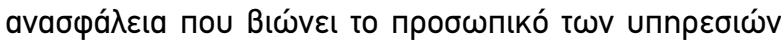

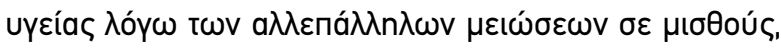

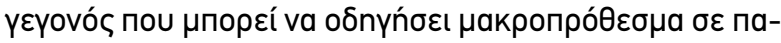

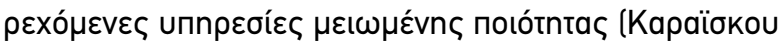
kat ouv 2012).

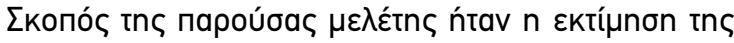

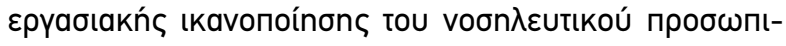

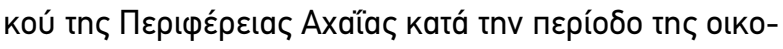
voutkńs kpíons.

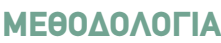

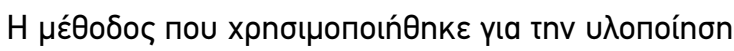

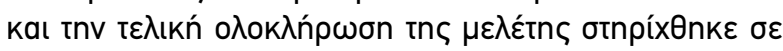

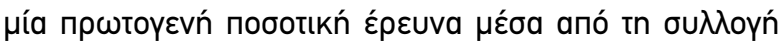

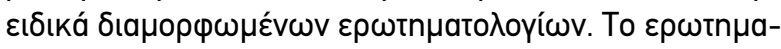

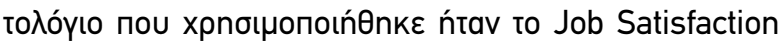

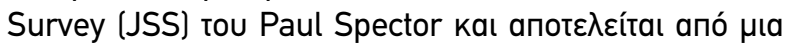

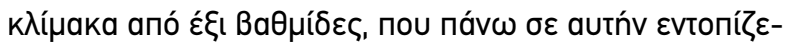

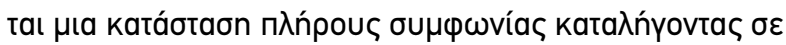

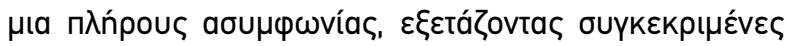

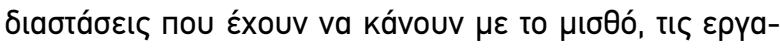

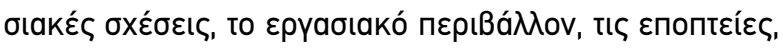

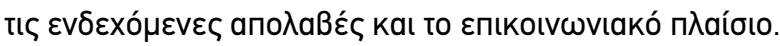

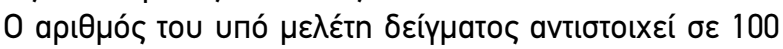

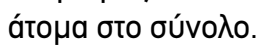

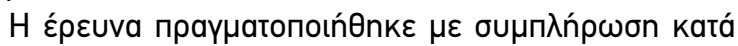

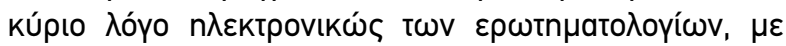

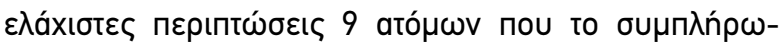

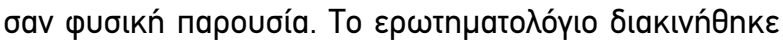

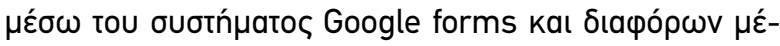

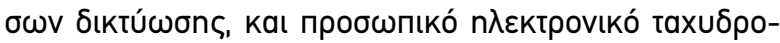
$\mu \varepsilon i ́$,

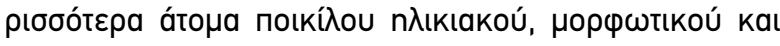

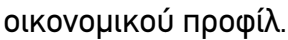

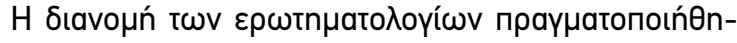

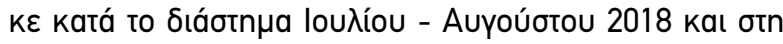

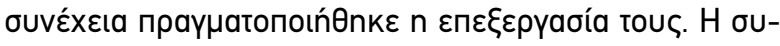

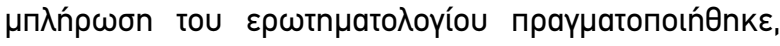

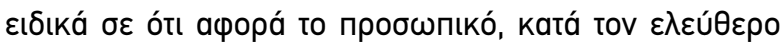

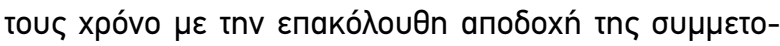

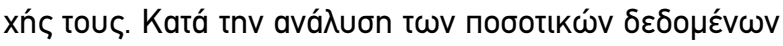

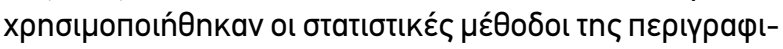

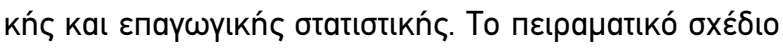

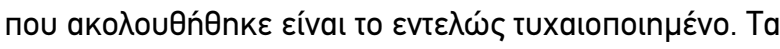

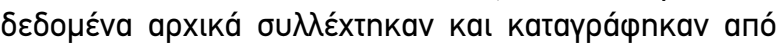

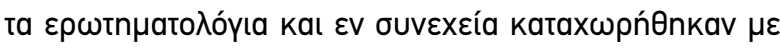

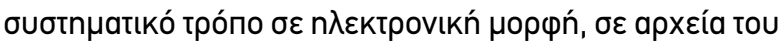

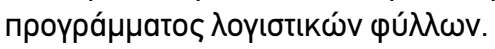

\section{AПОTЕАЕЕMATA}

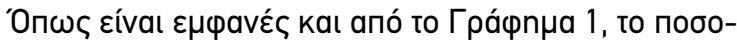

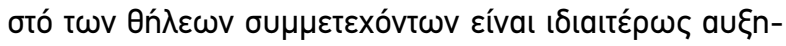

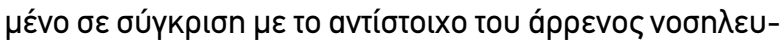

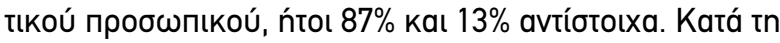

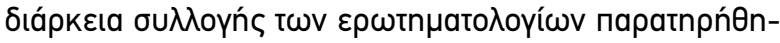

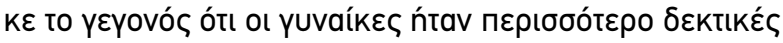

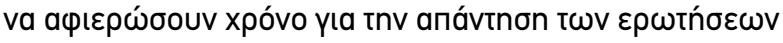

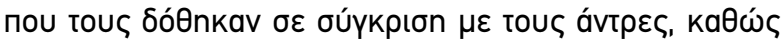

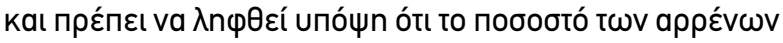

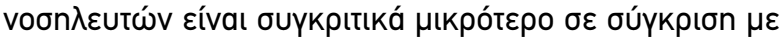

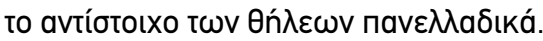

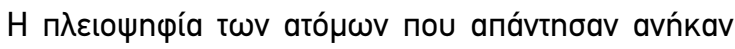

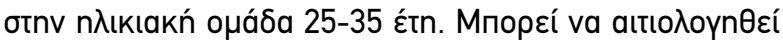

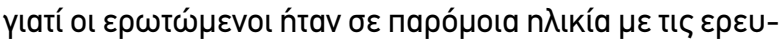

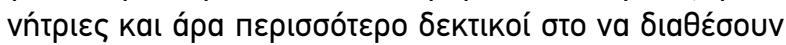

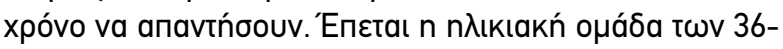

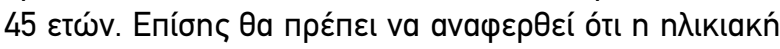

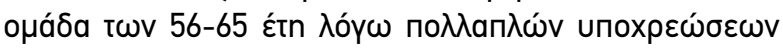

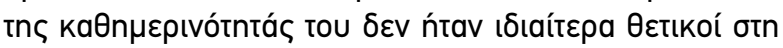

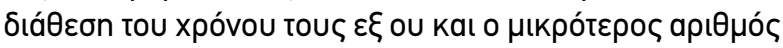

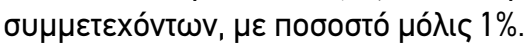

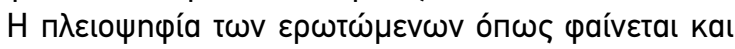

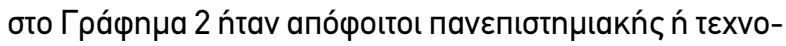




\section{EPEYNHTIKA APOPA}

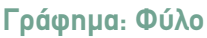

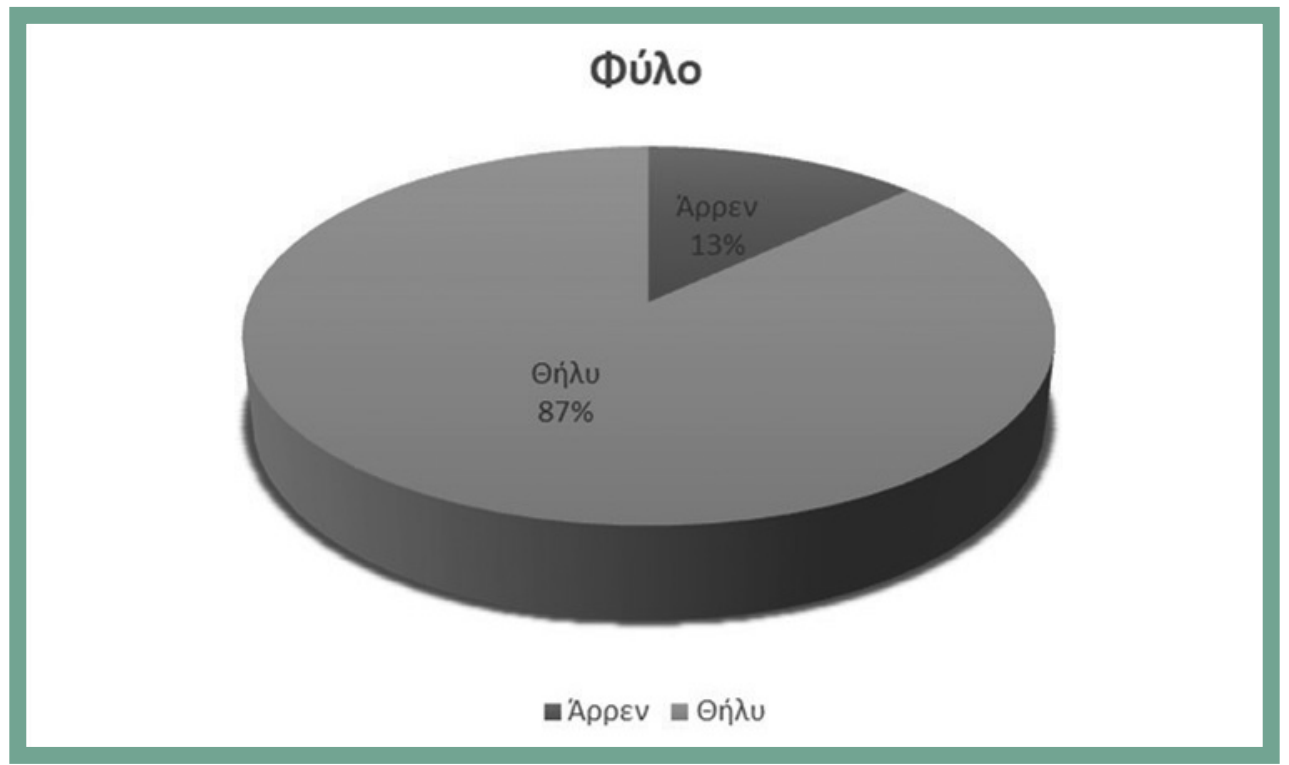

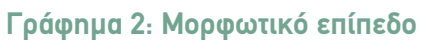

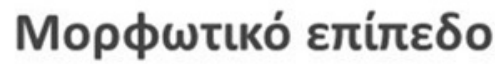

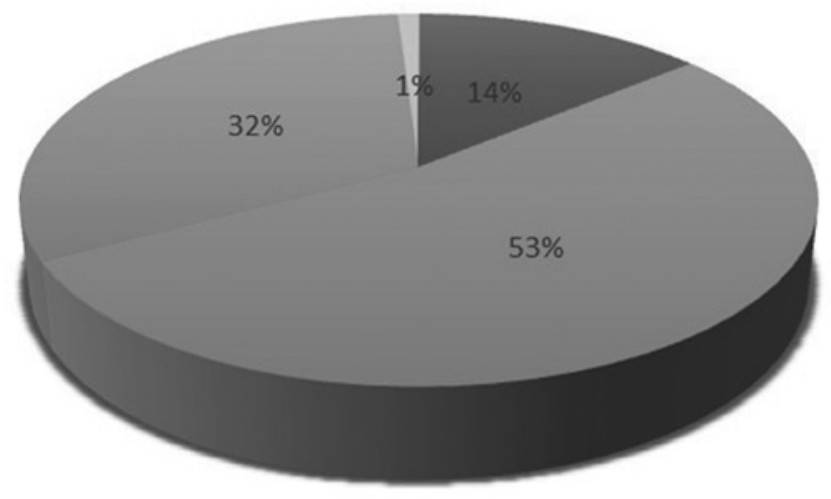

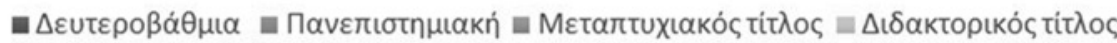

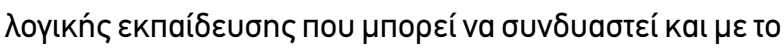

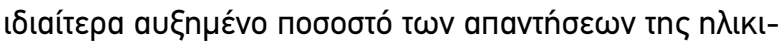

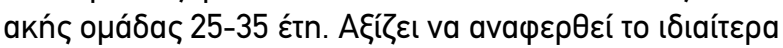

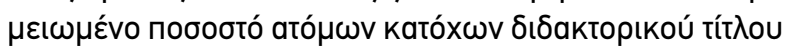

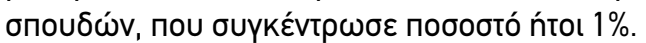

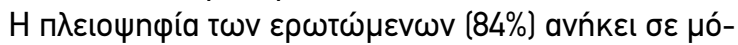

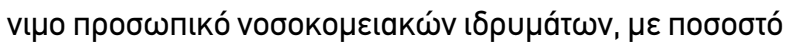

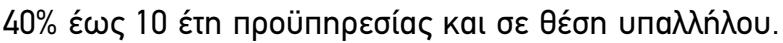

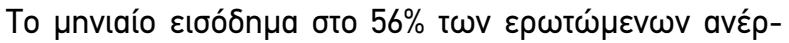

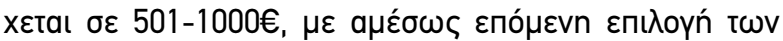

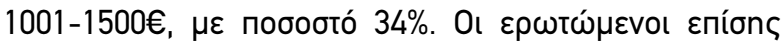

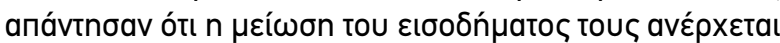

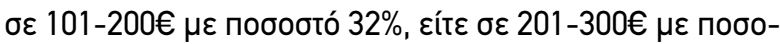
бтó $37 \%$.

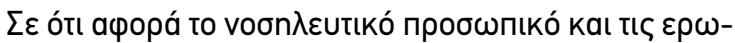

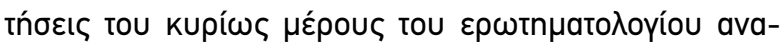

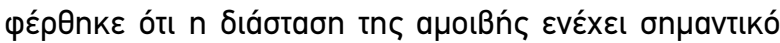

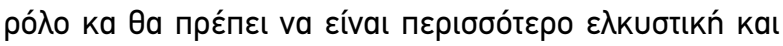




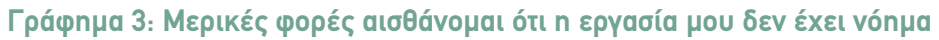

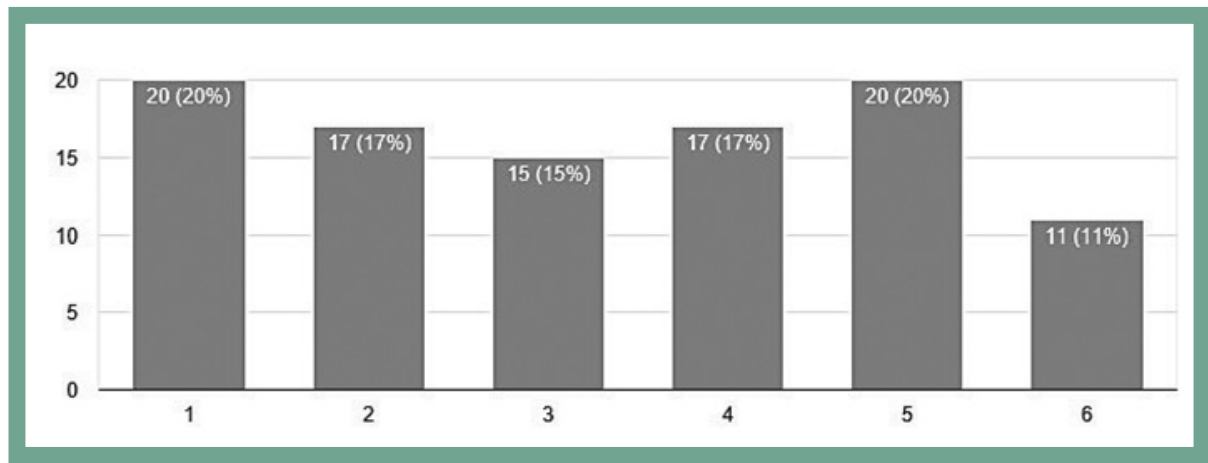

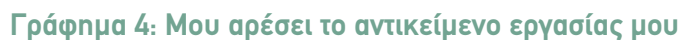

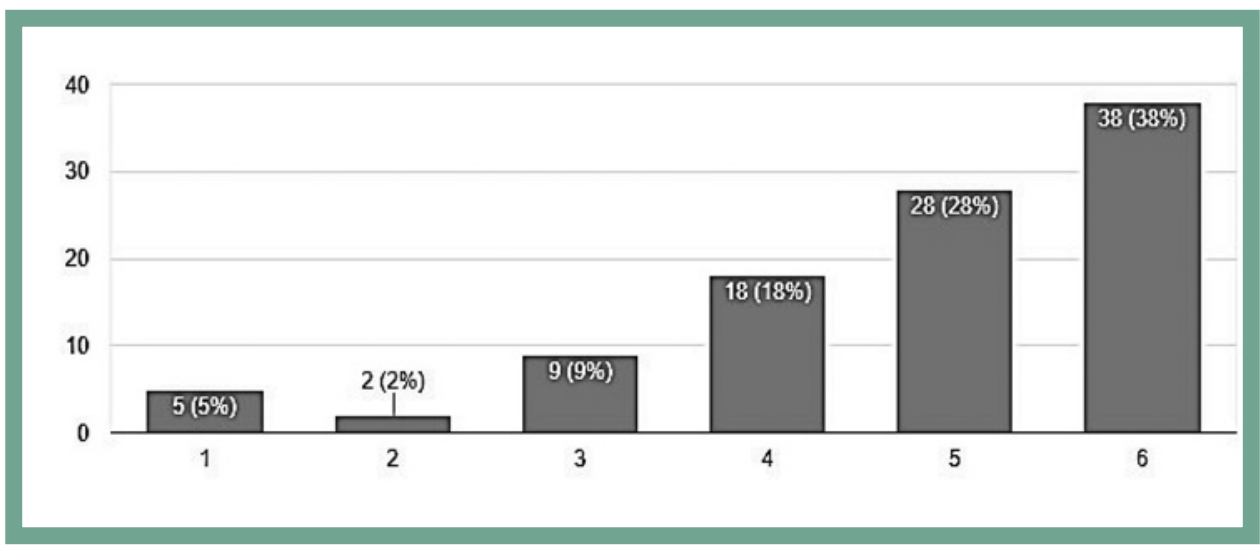

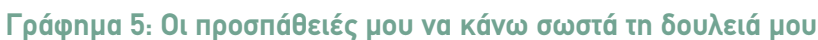

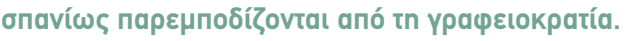

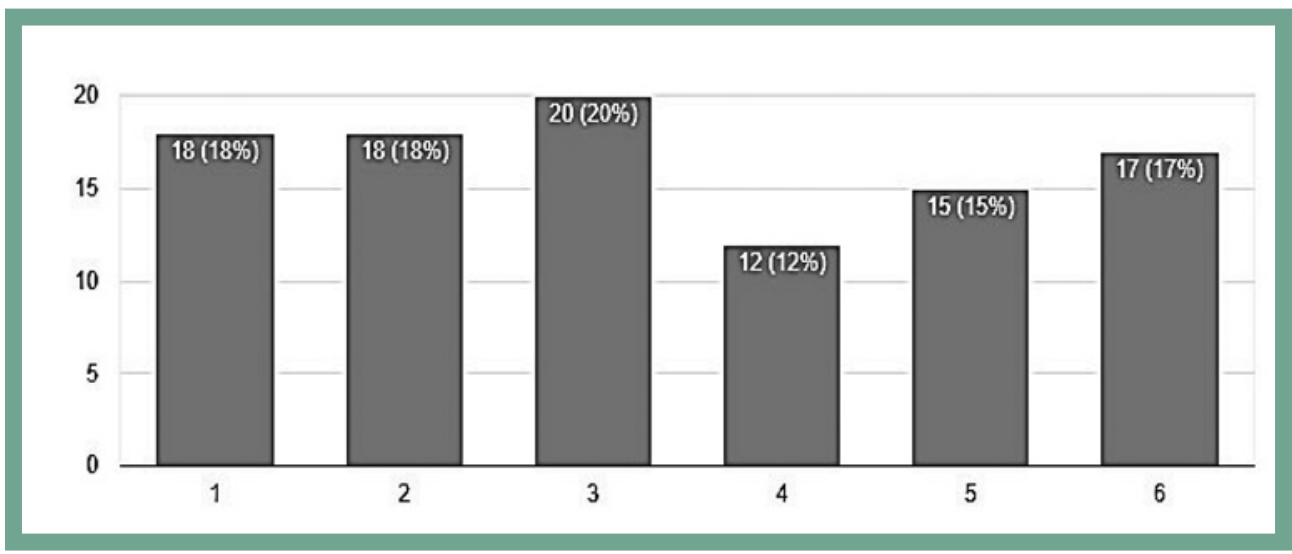

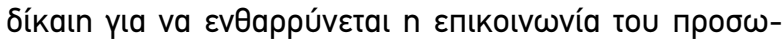

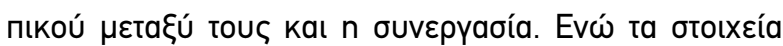

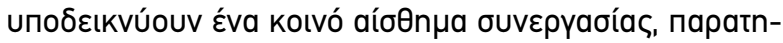

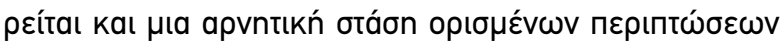

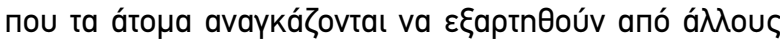

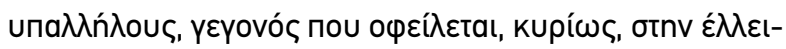

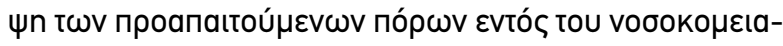

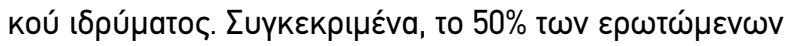




\section{EPEYNHTIKA APӨPA}

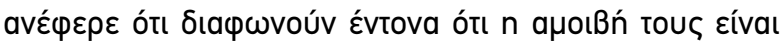

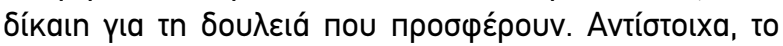

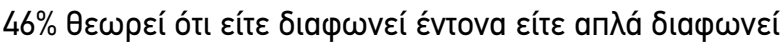

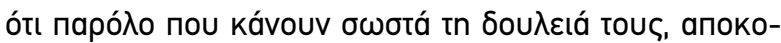

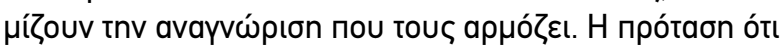

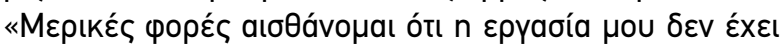

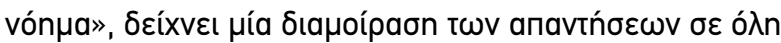

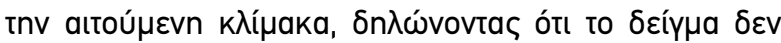

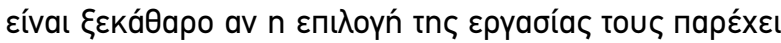

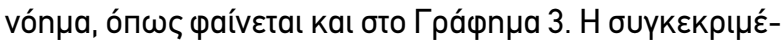

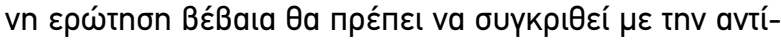

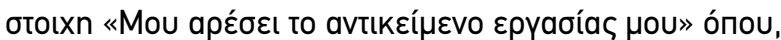

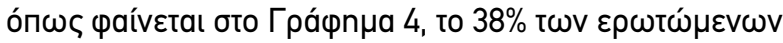

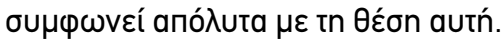

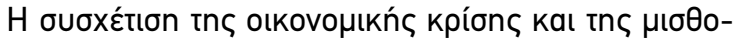

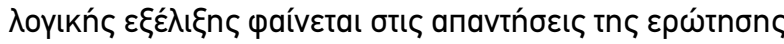

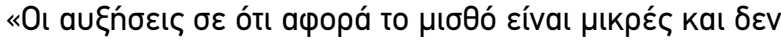

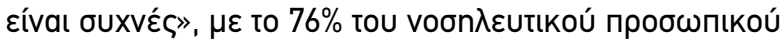

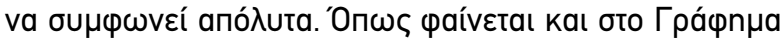

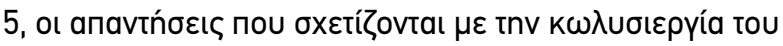

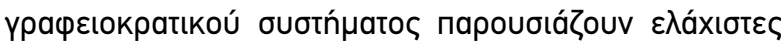

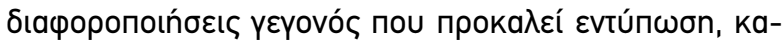

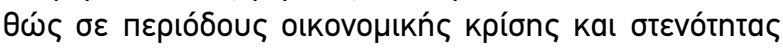

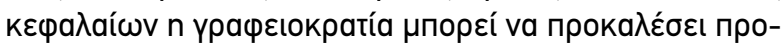

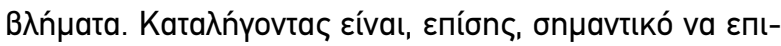

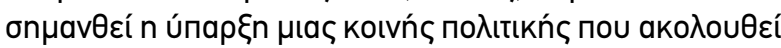

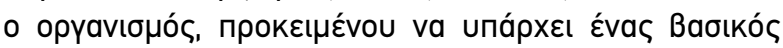

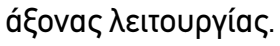

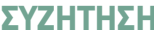

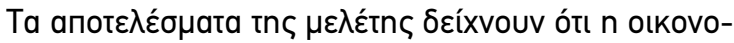

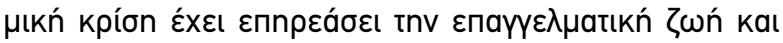

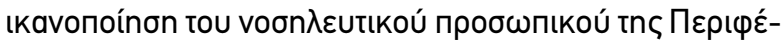

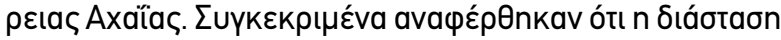

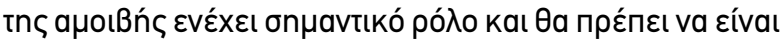

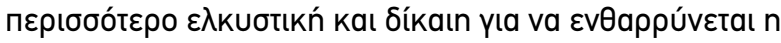
$\varepsilon \Pi ı$ เ

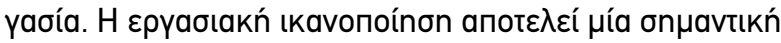

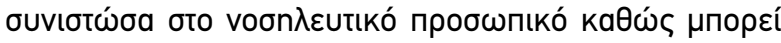

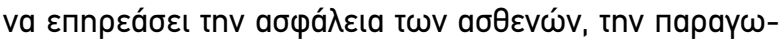

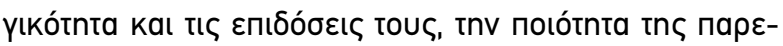

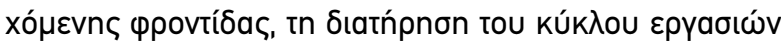

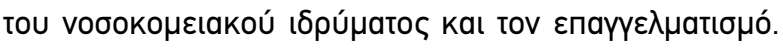

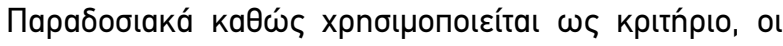

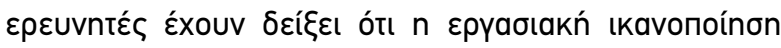

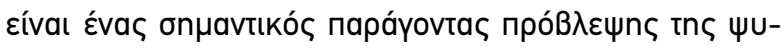

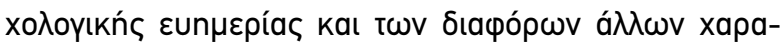

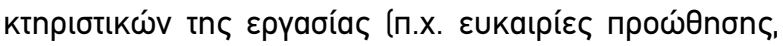

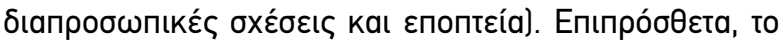

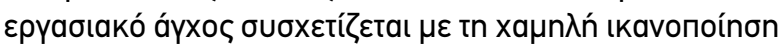

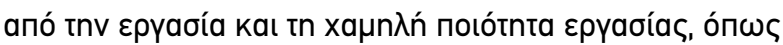

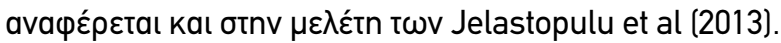

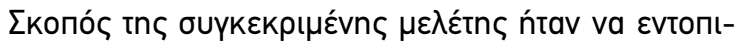

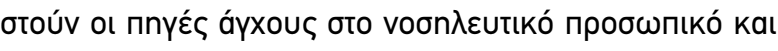

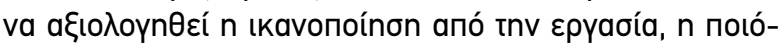

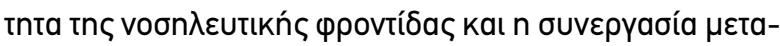

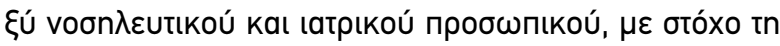

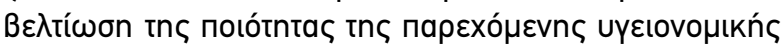

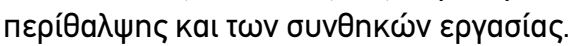

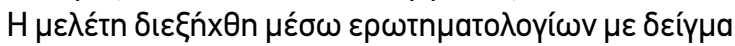

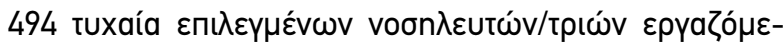

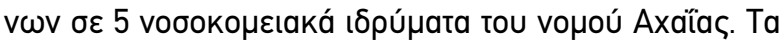

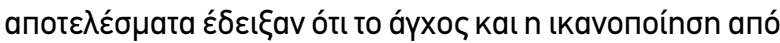

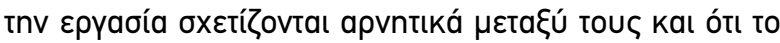

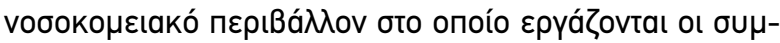

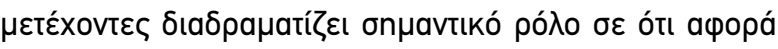

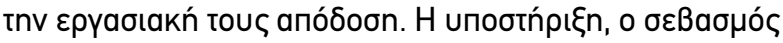

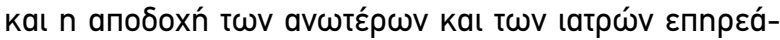

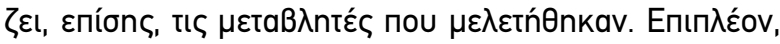

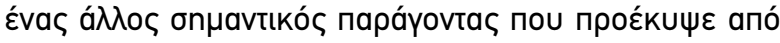

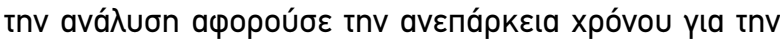

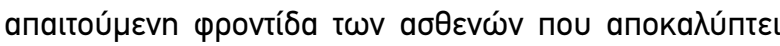

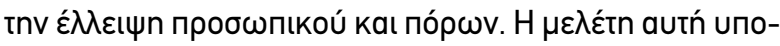

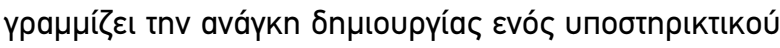

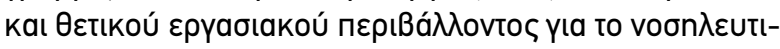
kó проб

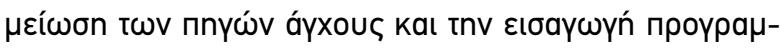

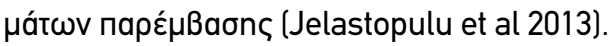

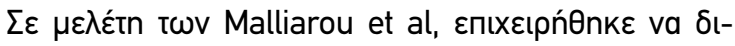

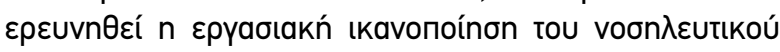

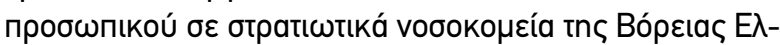

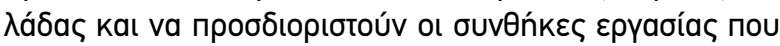

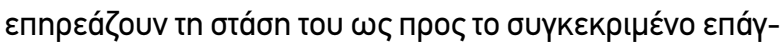

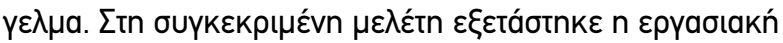

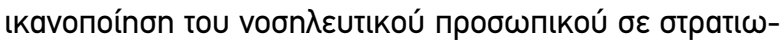

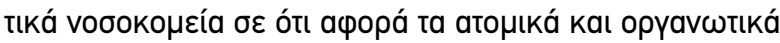

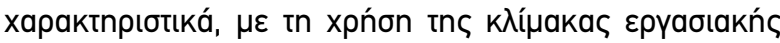

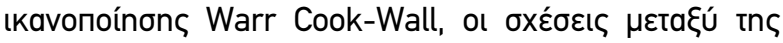

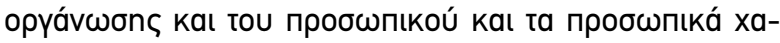

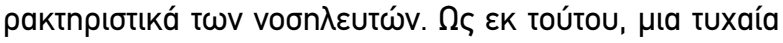

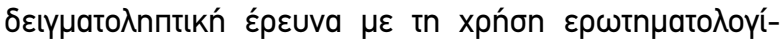

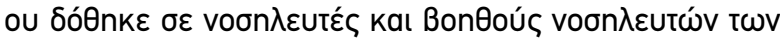

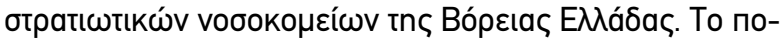

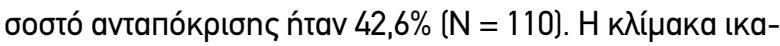

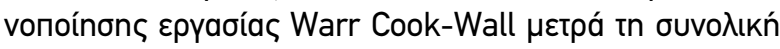

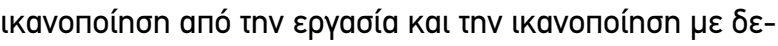

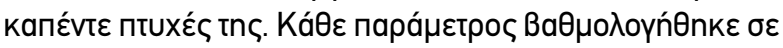

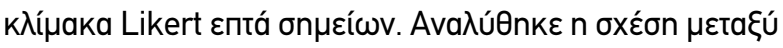

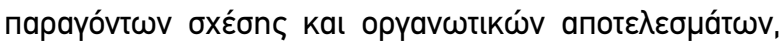

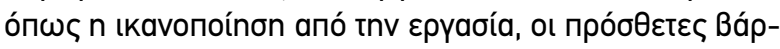

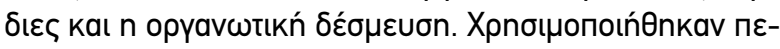

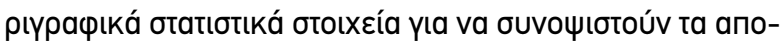

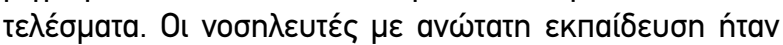

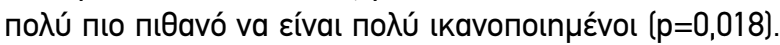

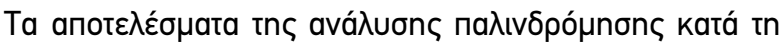

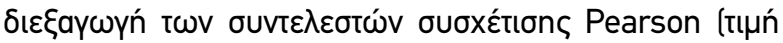




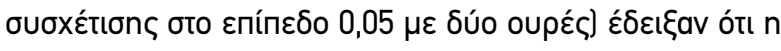
$\mu \varepsilon i ́ \omega \sigma n$ tnc tKavonoínon

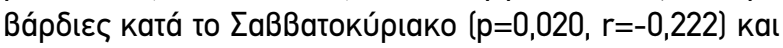

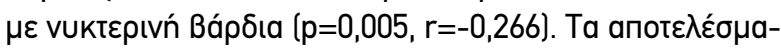

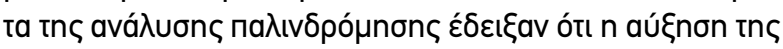

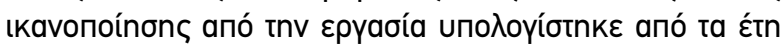

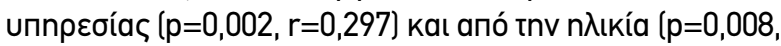

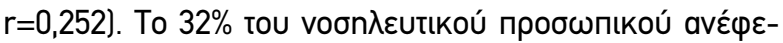

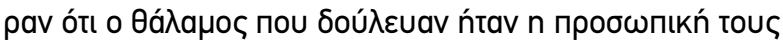

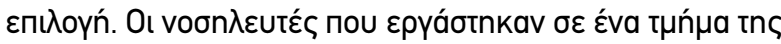

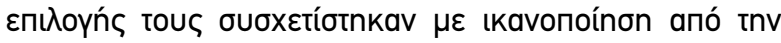

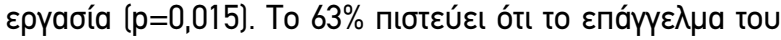

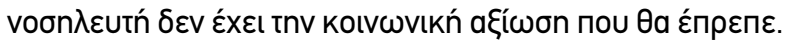

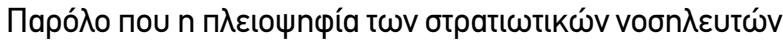

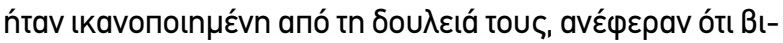

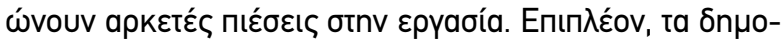

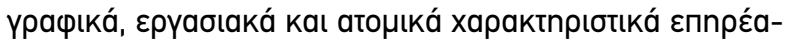

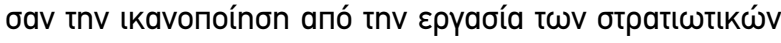

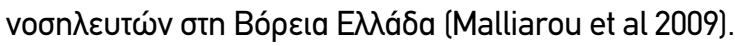

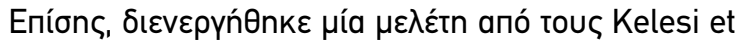

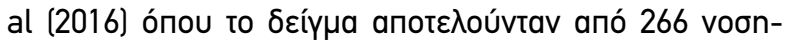

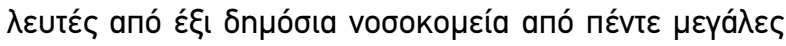

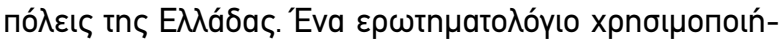

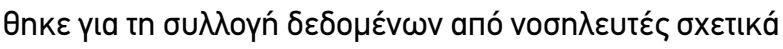
$\mu \varepsilon$ tnv Ikavonoínon anó tnv epyaoía touç. H ikavonoínon

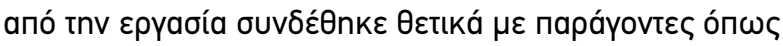

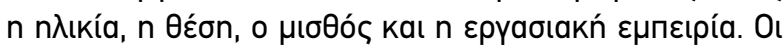

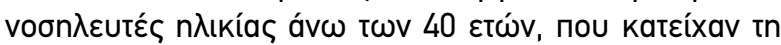

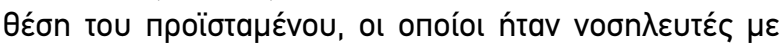

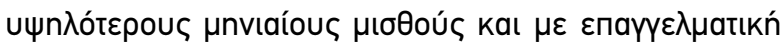

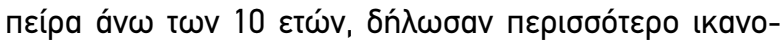

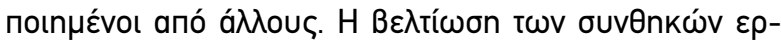

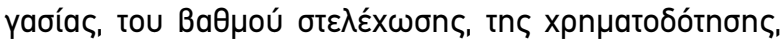

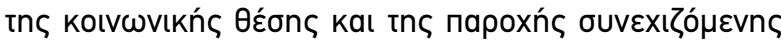

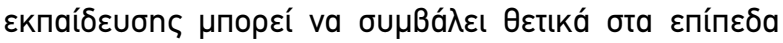

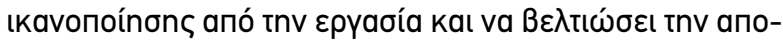

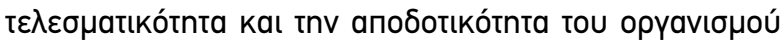
(Kelesi et al 2016).

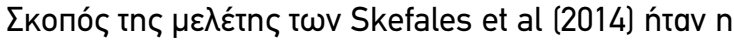

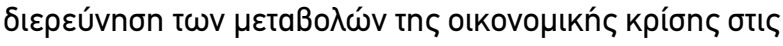

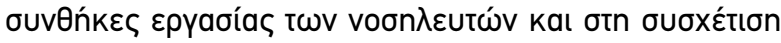

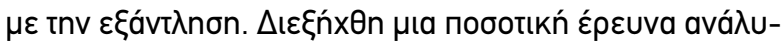

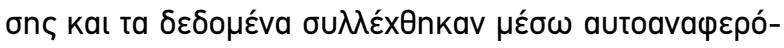

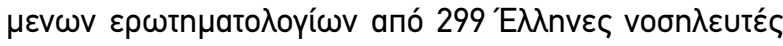

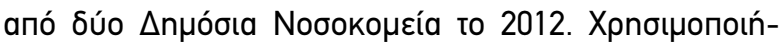

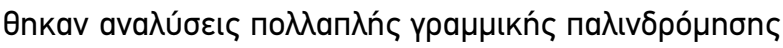

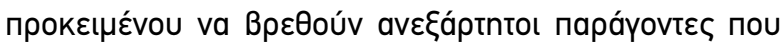

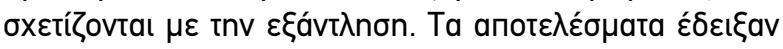

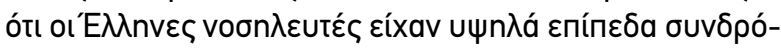

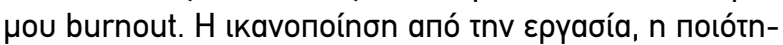

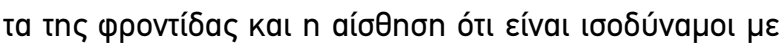

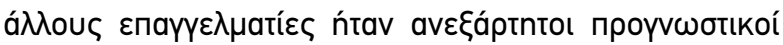

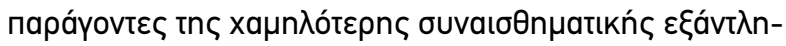

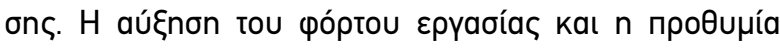

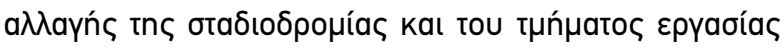

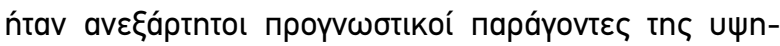

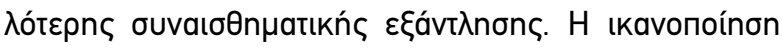

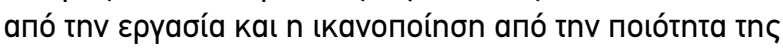

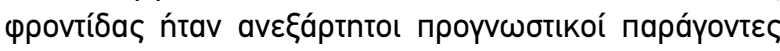

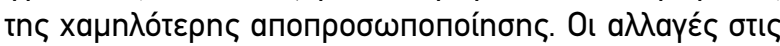

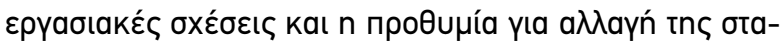

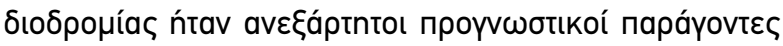

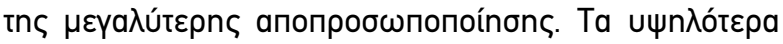

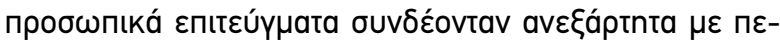

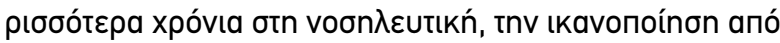

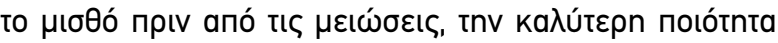

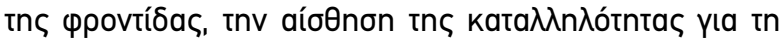

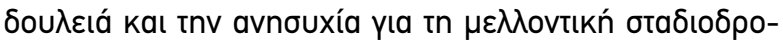

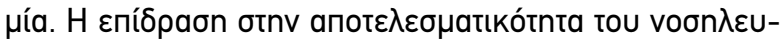

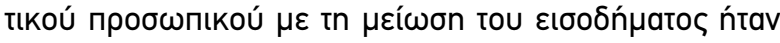

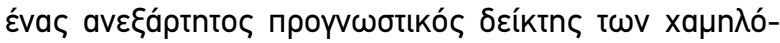

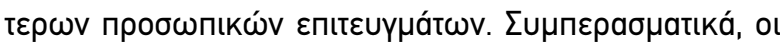

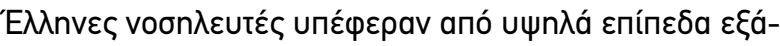

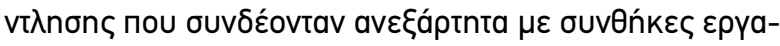

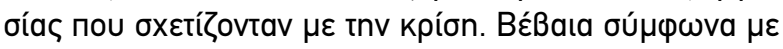

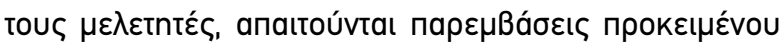

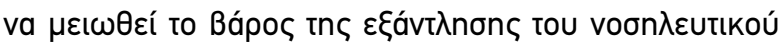
пробшпıкоú (Skefales et al 2014).

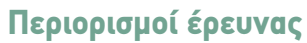

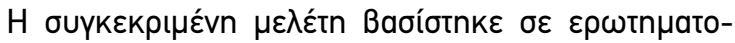

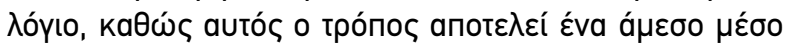

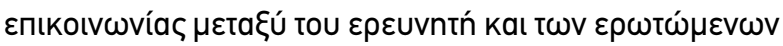

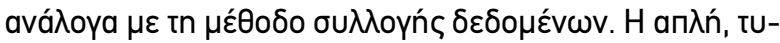

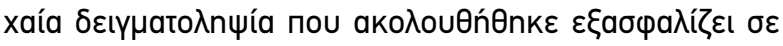

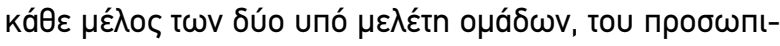

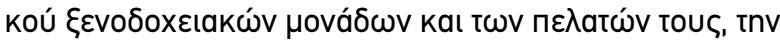

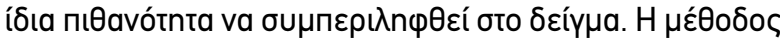

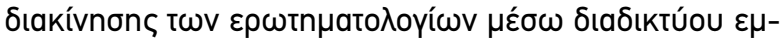

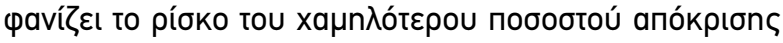

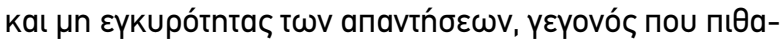

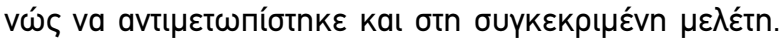

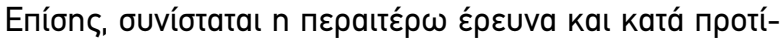

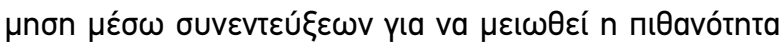

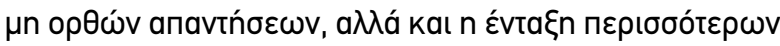

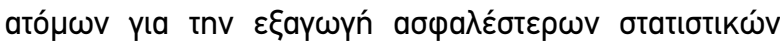

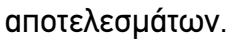

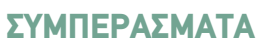

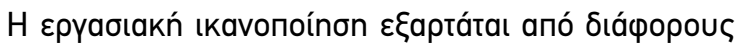

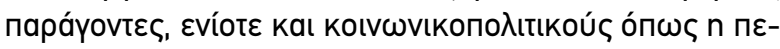

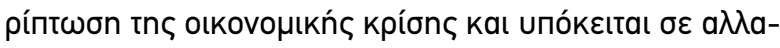

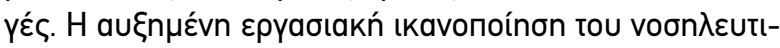

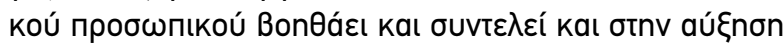

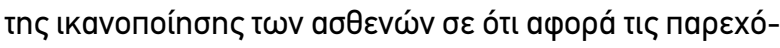

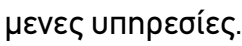




\section{ВІВАІОГРАФІА}

Doef (van der) M., Mbazzi F.B. \& Verhoeven C. (2012). Job conditions, job satisfaction, somatic complaints and burnout among East African nurses. Journal of Clinical Nursing 21(11-12): 1763-1775.

Faragher E.B., Cass M. \& Cooper C. (2005). The relationship between job satisfaction and health: A meta-analysis. Occupational and Environmental Medicine 62:105-112.

Gouzou M., Karanikola M., Lemonidou C., Papathanasoglou E. \& Giannakopoulou M. (2015). Measuring professional satisfaction and nursing workload among nursing staff at a Greek Coronary Care Unit. Rev Esc Enferm USP 49(Esp):15-21.

Jelastopulu E., Tsouvaltzidou T., Vangeli E., Messolora F., Detorakis J. \& Alexopoulos E. (2013). Self-reported sources of stress, job satisfaction and quality of care in professional hospital nurses in West-Greece. Nursing and Health 1(1):1-9.

Kelesi M., Fasoi G., Papageorgiou D., Tsaras K., Kaba E., Stavropoulou A., Polykandriotis T. \& Vlachou E. (2016). An Investigation Of Factors Determining The Level Of Job Satisfaction Among Nurses In Six General Public Hospitals In Greece. International Journal Of Medical Science And Clinical Inventions 3(3): 1705-1716.

Locke E. (1976). The nature and causes of job satisfaction, Dunnette M.(ed): Handbook of industrial and organizational psychology,

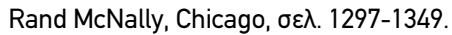

Locke E.A. (1969) What is job satisfaction? Organizational Behavior and Human Performance 4 (4): 309-336.
Malliarou M., Moustaka E. \& Konstantinidis T. (2009). Job satisfaction among nurses staff in military health care of Northern Greece. Balkan Military Medical Review 12:63-71.

Platis Ch., Reklitis P. \& Zimeras S. (2015) Relation between job satisfaction and job performance in healthcare services. Procedia - Social and Behavioral Sciences 175: 480 - 487.

Schaffer R.H. (1953). Job satisfaction as related to need satisfaction in work. Psychological Monographs: General and Applied 67(14): $1-29$

Skefales A., Plakas S., Fouka G., Zakka Goni M., Vassiliadou M. \& Bergiannaki J.D. (2014). Burnout and Its Association with Working Conditions among Greek Hospital Nurses in a Time of Financial Crisis. Open Journal of Nursing 4: 548-563.

Spector P. (1997). Job satisfaction: Application, assessment, causes and consequences. Sage publications Inc.

Visser M.R.M. (2003). Stress, satisfaction and burnout among Dutch medical specialists. Canadian Medical Association Journal168: 271-275.

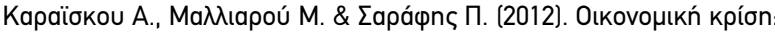

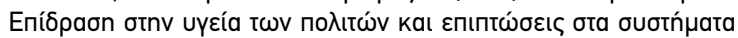

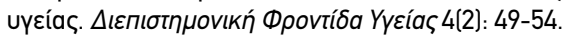

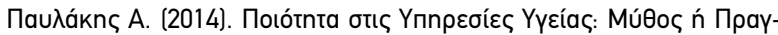

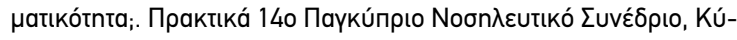
прос. 


\title{
Healthcare personnels' job satisfaction during the economic crisis in Achaia Region
}

\author{
Zoe Aggelopoulou', Maria Tzoumanika² \\ 1. RN, MSc, Children Intensive Care Unit, University General Hospital of Patras \\ 2. RN, Paediatrics Specialty, Neonatal Intensive Care Unit, University General Hospital of Patras
}

\begin{abstract}
Introduction: The economic crisis of recent years has inevitably led to an increase in unemployment due to the decline in the economic power of the labor market. As a consequence, discomfort and consequently a decrease in quality of life is spotted in the wider work environment, which depends on both endogenous and exogenous factors.

Aim: The impact of the economic crisis on the job satisfaction of nursing staff in the Region of Achaia. Methods: The present study has looked into factors such as salary, labor relations, supervision, potential earnings, the general work environment and the communication context. The Paul Spector Job Satisfaction Survey (JSS) was used, consisting of a 6-step scale from full agreement of the respondent to complete discrepancy. The sample size was 100 persons, the majority of which completed the questionnaire electronically, except from nine individuals who completed the questionnaire by hand. Quantitative data analysis was used in a randomized experimental design regarding descriptive and inductive statistics.

Results: The majority of the respondents were mainly females (87\%). The 25-35 years old age group was the main age group of the respondents. Regarding the question about their remuneration, $50 \%$ of respondents described it as unfair with regards to their work, which was in line with the $39 \%$ of those who generally felt that their work was not well appreciated. Furthermore, the majority of the sample felt less likely to be promoted.

Conclusions: The conclusions drawn from the study showed that the job satisfaction of the nursing staff in the Achaia Region has been negatively affected by the economic crisis. Significant reference was made by respondents to their salary, as they believed that they should be modified to reflect a fairer recognition of the difficulty and importance of their work, and to enhance the sense of collaboration and coexistence through more efficient communication.
\end{abstract}

Key-words: Financial crisis, job satisfaction, nursing staff. 